\title{
Gluons in a Color-Neutral Nucleus ${ }^{1}$
}

\author{
Gregory Mahlon \\ Department of Physics, McGill University \\ 3600 University St., Montréal, QC, Canada H3A 2T8
}

\begin{abstract}
We improve the McLerran-Venugopalan model $[1,2]$ by introducing a charge-density correlation function which is consistent with the observation that nucleons carry no net color charge. The infrared divergence in the transverse coordinates that was present in the McLerran-Venugoplan model is eliminated by the enforcement of color neutrality.
\end{abstract}

The problem of extracting first principles predictions from the theory of strong interactions, quantum chromodynamics (QCD), is notoriously difficult, largely because of the nonlinearity of the theory. The quanta of the gauge field, the gluons, themselves carry color charge and so serve as a source of additional gluons. Thus, any regime in which it is possible to actually compute some physical observable within the framework of QCD is of great interest.

Such is the case with the McLerran-Venugopalan (MV) model [1,2]. What McLerran and Venugopalan realized is that for very large nuclei at very small values of the longitudinal momentum fraction, the number of color charges participating in the generation of the $\mathrm{QCD}$ vector potential is large. In this situation, the gluon number density may be approximated to lowest order by solving the classical YangMills equations in the presence of the (classical) source generated by the valence quarks. To actually extract the gluon number density, we must average over the sources to obtain the two point correlation function for the vector potential. This approximation may be systematically improved by including the quantum corrections. The correlation function derived in Ref. [2] is highly infrared divergent at large transverse distances. In this talk, we will show that this difficulty may be ameliorated by forcing the nucleons to obey a color-neutrality condition [3].

Before proceeding with the main part of this talk, it is necessary to say a few words about our notation and conventions. We elect to work with light-cone coordinates, defined by $x^{ \pm} \equiv\left(x^{0} \pm x^{3}\right) / \sqrt{2}$. The components of a 4 -vector will be written as $x=\left(x^{+}, x^{-}, \vec{x}\right)$, with vectors in the two-dimensional (transverse) subspace written with arrows. We choose a metric with the signature $(-,+,+,+)$. Consequently,

1) Talk presented at MRST'99: High Energy Physics at the Millenium, Carleton University, Ottawa, Ontario, Canada, May 10-12, 1999. 


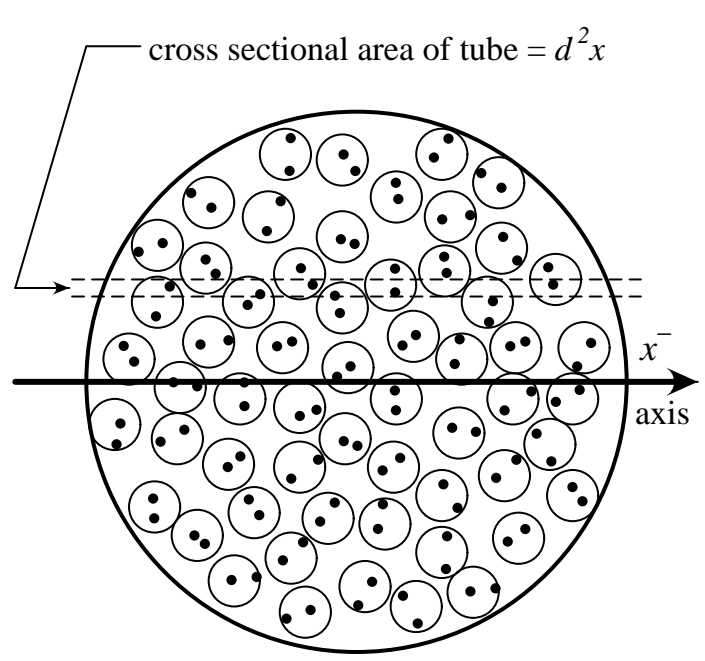

FIGURE 1. A cartoon of a very large nucleus in its rest frame. In the labatory frame, Lorentz contraction causes all of the quarks and nucleons to pile up at essentially the same value of $x^{-}$.

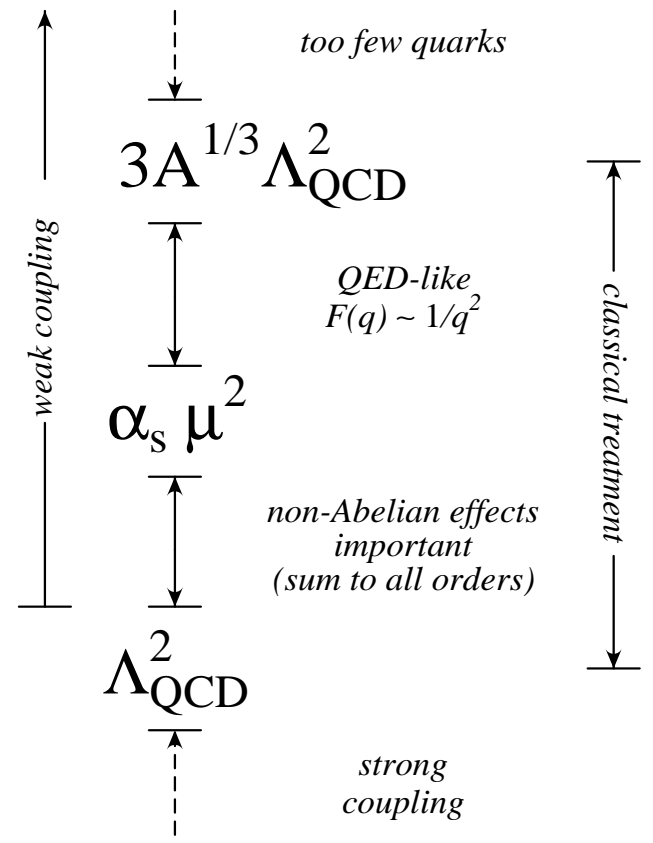

FIGURE 2. Map of transverse momentum scales in the McLerran-Venugopalan model.

we have $x_{ \pm}=-x^{\mp}$, and a dot product which $\operatorname{reads} x \cdot q \equiv-x^{+} q^{-}-x^{-} q^{+}+\vec{x} \cdot \vec{q}$. We will work in the light-cone gauge, $A^{+} \equiv 0$, where the intuitive parton model is realized [4].

Within the MV model [1,2], the Lorentz-contracted nucleus is treated as a pancake of color charge coming from the valence quarks. For a large nucleus, this means that a tube of cross-sectional area $d^{2} \vec{x}$ will intercept a large number of valence quarks (see Fig. 1). If we restrict ourselves to small longitudinal momentum fractions, then all of the quarks in the tube effectively overlap. Because a large number of quarks contribute, the color charge is in a high-dimensional representation of the group, and may be approximated by a classical charge density.

To compute the gluon number density, we begin by solving the classical YangMills equation for the vector potential $A^{\mu}$ as a function of the color charge density $\rho$. Next, the usual quantum mechanical average is approximated by an average over an ensemble of nuclear charge distributions to compute two-point correlation functions of the vector potential, $\left\langle A_{i}^{a}\left(x^{-}, \vec{x}\right) A_{j}^{b}\left(x^{\prime-}, \vec{x}^{\prime}\right)\right\rangle$. These correlation functions contain the gluon number operator; hence, they are be connected to the gluon number density. The result of the averaging process depends on the form chosen for the two-point charge density correlation function $\left\langle\rho^{a}\left(x^{-}, \vec{x}\right) \rho^{b}\left(x^{\prime-}, \vec{x}^{\prime}\right)\right\rangle$. Finally, we obtain the gluon number density by performing the appropriate Fourier transform of the position space correlator.

Under what conditions is it legitimate to use the classical approximation we have just described? Schematically, the situation is as illustrated in Fig. 2. If the typical transverse momenta being considered is too small (below $\Lambda_{\mathrm{QCD}}$ ), then we are in 
the stong coupling regime, where we have no reason to expect classical methods to be valid. At larger transverse momentum scales, we have weak coupling. However, we cannot go to too large a transverse momentum, or else the requirement that we have a large number of quarks contributing in each patch $d^{2} \vec{x}$ of nucleus no longer holds: the quantum graininess begins to become important. At the upper end of where the classical approximation is valid, the solution is QED-like, with a distribution function which is proportional to $1 / \vec{q}^{2}$. Somewhere in between is a cross-over scale where the non-Abelian terms in the equations of motion become important, and we require a solution which is summed to all orders.

We now turn to an examination of the basic form of the classical solution, using the situation in electrodynamics as our guide. Consider a point charge $e$ moving at the speed of light down the $z$ axis. In this situation, the current is

$$
J^{+}=e \delta\left(x^{-}\right) \delta^{2}(\vec{x}) ; \quad J^{-}=0 ; \quad \vec{J}=0 .
$$

Unlike in the (more familiar) Lorentz gauge where the $\mu$ th component of the current generates the $\mu$ th component of the vector potential, in light cone gauge only the transverse components of $A^{\mu}$ are non-zero:

$$
A^{i}(x)=\frac{e}{2 \pi} \Theta\left(x^{-}\right) \frac{x^{i}}{\vec{x}^{2}}
$$

This vector potential corresponds to the non-vanishing field tensor components

$$
F^{+i}=-\frac{e}{2 \pi} \delta\left(x^{-}\right) \frac{x^{i}}{\vec{x}^{2}} .
$$

These components of the field tensor correspond to transverse electric and magnetic fields of equal strength. There are no longitudinal fields in this limit. An observer sitting at some fixed position $\left(\vec{b}, x^{3}\right)$ would see no fields except at the instant when the charge made its closest approach, when a $\delta$-function pulse would be seen. The magnitude of the pulse would be proportional to $1 / b$. The (unobservable) vector potential, which was zero before the passage of the charge, takes on a non-zero value for all times afterward. Since the fields vanish at these times, the late-time vector potential may be thought of as some particular gauge transformation of the vacuum.

The overall features of the above description continue to hold when we switch to QCD, although the details are slightly altered by the presence of the non-Abelian terms in the field equations. The net effect of these terms is to color-rotate the source in a complicated fashion. Nevertheless, the chromoelectric and chromomagnetic fields are non-zero only at the instant of closest approach by the charge, and the vector potential switches from one gauge transform of the vacuum to a different one at this instant.

We now outline the method used to compute the correlation function $\left\langle A_{i}^{a}\left(x^{-}, \vec{x}\right) A_{j}^{b}\left(x^{\prime-}, \vec{x}^{\prime}\right)\right\rangle$. Full details of this part of the calculation are found in 
Ref. [2]. Essentially, what one does is to expand the vector potential in "powers" of the charge density $\rho$, and perform the averaging by doing all possible pairwise contractions using

$$
\left\langle\rho^{a}\left(x^{-}, \vec{x}\right) \rho^{b}\left(x^{\prime-}, \vec{x}^{\prime}\right)\right\rangle=\delta^{a b} \mu^{2}\left(x^{-}\right) \delta\left(x^{-}-x^{-}\right) \mathcal{D}\left(\vec{x}-\vec{x}^{\prime}\right) .
$$

In this expression $\mu^{2}\left(x^{-}\right)$is the color charge squared per unit area per unit thickness (i.e. per unit $x^{-}$). We set up the calculation with a non-zero nuclear thickness to avoid ambiguities in the commutator terms of the Yang-Mills equations which would arise if we let $\rho \sim \delta\left(x^{-}\right)$exactly. Refering back to Fig. 1, we see that the quarks in our "tube" of color charge typically come from different nucleons. Thus, we expect them to be uncorrelated, hence the dependence $\delta\left(x^{-}-x^{\prime-}\right)$ in Eq. (4). The transverse dependence of the charge denisty correlator is given by $\mathcal{D}\left(\vec{x}-\vec{x}^{\prime}\right)$. In Ref. [2], it is argued that this should also be a delta-function, since we are restricted to length scales $\lesssim \Lambda_{\mathrm{QCD}}^{-1}$. However, as we shall see, doing so is not consistent with color neutral nucleons, and leads to severe infrared divergences in the correlation funtion.

After doing all of the contractions, we resum the series to obtain the master formula

$$
\left\langle A_{i}^{a}\left(x^{-}, \vec{x}\right) A_{j}^{b}\left(x^{\prime-}, \vec{x}^{\prime}\right)\right\rangle=\frac{\delta^{a b}}{N_{c}} \frac{\partial_{i} \partial_{j}^{\prime} L\left(\vec{x}-\vec{x}^{\prime}\right)}{L\left(\vec{x}-\vec{x}^{\prime}\right)}\left[e^{N_{c} \chi\left(x^{-}, x^{\prime-}\right) L\left(\vec{x}-\vec{x}^{\prime}\right)}-1\right] .
$$

Eq. (5) depends on two new functions. The first, $\chi\left(x^{-}, x^{\prime-}\right)$, measures the amount of charge in those layers of the source which have already passed both of the points which we are comparing:

$$
\chi\left(x^{-}, x^{\prime-}\right) \equiv \int_{-\infty}^{\min \left(x^{-}, x^{-}\right)} d \xi^{-} \mu^{2}\left(\xi^{-}\right)
$$

The appearance of this function may be understood by recalling that the value of the vector potential depends on whether or not the charge has yet reached its point of closest approach. Although the range of integration in (6) extends to $x^{-}=-\infty$, in practice this is cut off by the form of $\mu^{2}\left(x^{-}\right)$, which for a pancake-shaped charge distribution, should be non-zero only in a relatively small range near the value of $x^{-}$that corresponds to the position of the nucleus.

The second new function appearing in (5) is given by

$$
\begin{aligned}
L\left(\vec{x}-\vec{x}^{\prime}\right) \equiv \int d^{2} \vec{\xi} \int d^{2} \vec{\xi}^{\prime} \mathcal{D}\left(\vec{\xi}-\vec{\xi}^{\prime}\right)\left[G(\vec{x}-\vec{\xi}) G\left(\vec{x}^{\prime}-\vec{\xi}^{\prime}\right)\right. & \\
& \left.-\frac{1}{2} G(\vec{x}-\vec{\xi}) G\left(\vec{x}-\vec{\xi}^{\prime}\right)-\frac{1}{2} G\left(\vec{x}^{\prime}-\vec{\xi}\right) G\left(\vec{x}^{\prime}-\vec{\xi}^{\prime}\right)\right],
\end{aligned}
$$

where

$$
G\left(\vec{x}-\vec{x}^{\prime}\right) \equiv \frac{1}{4 \pi} \ln \left(\frac{\left|\vec{x}-\vec{x}^{\prime}\right|^{2}}{\lambda^{2}}\right)
$$


This Green's function is the inverse of the operator $\vec{\nabla}^{2}$ (in two dimensions). The lack of a scale in our theory produces an infrared divergence in this function, which is signalled by the appearance of an arbitrary length scale $\lambda$. Clearly, the infrared finiteness or lack thereof of $L\left(\vec{x}-\vec{x}^{\prime}\right)$ is intimately related to the form chosen for $\mathcal{D}\left(\vec{\xi}-\vec{\xi}^{\prime}\right)$. On the other hand, according to Eq. (7), $L\left(\vec{x}-\vec{x}^{\prime}\right)$ vanishes when $\vec{x}=\vec{x}^{\prime}$, i.e. in the ultraviolet. Thus, at very short distances the nonlinear terms in (5) drop out and the behavior of the correlation function is the same as if we had considered a purely Abelian theory instead.

If, as in Ref. [2], we take $\mathcal{D}\left(\vec{\xi}-\vec{\xi}^{\prime}\right)=\delta^{2}\left(\vec{\xi}-\overrightarrow{\xi^{\prime}}\right)$, we end up with

$$
L\left(\vec{x}-\vec{x}^{\prime}\right) \sim \frac{1}{\vec{\nabla}^{4}}\left[\delta^{2}\left(\vec{x}-\vec{x}^{\prime}\right)-\delta^{2}(0)\right] .
$$

Although the subraction term serves to remove the quadratic infrared singularity, a logarithmic divergence remains. Assuming that the arbitrary scale should be of order $\Lambda_{\mathrm{QCD}}$ on physical grounds, the authors of Ref. [2] obtain

$$
\left\langle A_{i}^{a}\left(x^{-}, \vec{x}\right) A_{i}^{a}\left(x^{\prime-}, \vec{x}^{\prime}\right)\right\rangle=\frac{4\left(N_{c}^{2}-1\right)}{N_{c}\left|\vec{x}-\vec{x}^{\prime}\right|^{2}}\left[1-\left(\left|\vec{x}-\vec{x}^{\prime}\right|^{2} \Lambda_{\mathrm{QCD}}^{2}\right)^{\left(N_{c} / 16 \pi\right) \chi\left(x^{-}, x^{-}\right)\left|\vec{x}-\vec{x}^{\prime}\right|^{2}}\right] .
$$

This correlation function diverges like $\left(\vec{x}^{2}\right)^{\vec{x}^{2}}$ for large values of the separation. Of course, the bad behavior does not begin until the point $x \sim \Lambda_{\mathrm{QCD}}^{-1}$, the point where we begin to mistrust our calculation anyhow. Unfortunately, because of this divergence, the Fourier transform of Eq. (10) does not exist for any value of $\vec{q}$, real or imaginary. So it is difficult to see how to define the gluon number density more than qualitatively using this expression.

The resolution of this problem lies in recognizing the importance of enforcing the observation that nucleons, when observed on a large enough length scale, should be color neutral. A consequence of the Gaussian averaging employed in the MV model is that the average color charge vanishes, $\left\langle\rho^{a}\left(x^{-}, \vec{x}\right)\right\rangle=0$. However, we should also impose the (stronger) condition that

$$
\int d x^{-} d^{2} \vec{x} \rho^{a}\left(x^{-}, \vec{x}\right)=0
$$

for a nucleus-sized volume. If we integrate the charge density correlator (4) over all $\left(x, x^{\prime}\right)$ and apply (11) we obtain a constraint on the transverse portion of the correlator $\mathcal{D}$ :

$$
\int d^{2} \vec{x} \mathcal{D}(\vec{x})=0
$$

Any correlation function which satisfies Eq. (12) is compatible with color neutral nucleons. Such a correlation function must contain an intrinsic scale, i.e. the minimum transverse length scale for which (12) becomes true. When we compute $L(\vec{x})$, 
this scale will be imparted to the logarithms appearing in Eq. (7). This stongly suggests that the resulting correlation function determined from Eq. (5) will be infrared finite. Indeed, this is the case [3].

To illustrate the features of our improved treatment, we turn to a specific model of a large nucleus introduced by Kovchegov [5]. In this model, we view the nucleus as containing $A$ nucleons of radius $a$ distributed within a sphere of radius $R$. Each "nucleon" consists of a $q \bar{q}$ pair. By explicitly averaging over the allowed positions of the quarks, antiquarks, and nucleons we may explicitly compute the function $\mathcal{D}\left(\vec{x}-\vec{x}^{\prime}\right)$. We find that there are two types of terms. The first is generated when the position of two quarks (or two antiquarks) overlap. It is proportional to $\delta^{2}\left(\vec{x}-\vec{x}^{\prime}\right)$, precisely the form for $\mathcal{D}$ employed in Ref. [2]. The second kind of term enters in with opposite sign and corresponds to the situation when a quark overlaps an antiquark. It is proportional to a smooth function of the separation. Since the focus of Ref. [5] was on very short distances, this term was neglected relative to the delta-function contribution. However, at somewhat longer distance scales, it is precisely this additional contribution which is required to satisfy Eq. (12).

We have compiled a series of plots (Figs. 3-6) to aid in the comparison of our results using Kovchegov's model to the results of Ref. [2]. In addition to the uniform distribution of quarks, antiquarks, and nucleons employed by Kovchegov, we have also performed the averaging using Gaussian distributions. In preparing these plots, we have adjusted the nucleon size parameter $a$ and (for the MV result) $\Lambda_{\mathrm{QCD}}$ so that the corresponding correlation functions (5) match in the ultraviolet limit. This requires $a_{G}=0.464 a_{U}$ and $\Lambda_{\mathrm{QCD}}=1.44 a_{U}^{-1}$ where $a_{U}$ is the nucleon size parameter for the uniform quark distribution.

In Fig. 3 we have plotted the smooth part of the correlation function for the uniform and Gaussian cases, defined by writing $\mathcal{D}\left(\vec{x}-\vec{x}^{\prime}\right) \equiv \delta^{2}\left(\vec{x}-\vec{x}^{\prime}\right)-C\left(\vec{x}-\vec{x}^{\prime}\right)$. In both cases, the bulk of the contribution comes from separations less than $2 a .^{2}$ Therefore, in this and subsequent plots we have defined the dimensionless distance $X \equiv\left|\vec{x}-\vec{x}^{\prime}\right| /(2 a)$.

Next, we present Fig. 4, which shows the trace of the correlation function (5) for all three cases. The Fourier transform of this quantity is proportional to the gluon number density. The Gaussian curve, not surprisingly, has a Gaussian tail for $X \geq 2 a$, while the uniform curve vanishes identically in this region. On the other hand, the result from Ref. [2] runs off to $-\infty$ beyond $\Lambda_{\mathrm{QCD}}^{-1}$.

We come to the gluon number density in Fig. 5. To define the MV curve, we have followed the suggestion made in Ref. [6], and simply cut off the $\vec{x}$ integration at $x=\Lambda_{\mathrm{QCD}}^{-1}$. This is the source of the wiggles visible in Fig. 5. All three curves have the same overall shape, with a plateau at small values of $\vec{q}$ and a $1 / \vec{q}^{2}$ fall off at large $\vec{q}$. The number of zero momentum gluons clearly depends on how we have cut things off: the Gaussian model, which allows quarks to be (albeit with small probability) a large distance from the center of their nucleons has the most long

2) In fact, $C\left(\vec{x}-\vec{x}^{\prime}\right)$ vanishes identically for separations greater than $2 a$ when a uniform distribution is assumed. 


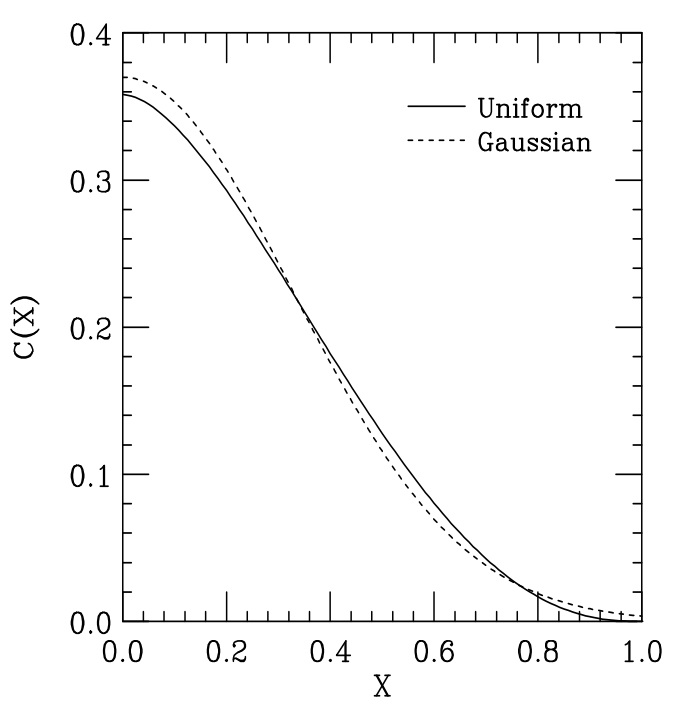

FIGURE 3. The smooth part of the two-point charge density correlation function in Kovchegov's model, defined by writing $\mathcal{D}(\vec{x}) \equiv \delta^{2}(\vec{x})-C(\vec{x})$. The nucleon size parameters have been chosen so that the resulting gluon number densities match in the ultraviolet limit. The two curves correspond to uniform and Gaussian distributions of the quarks inside the nucleons.

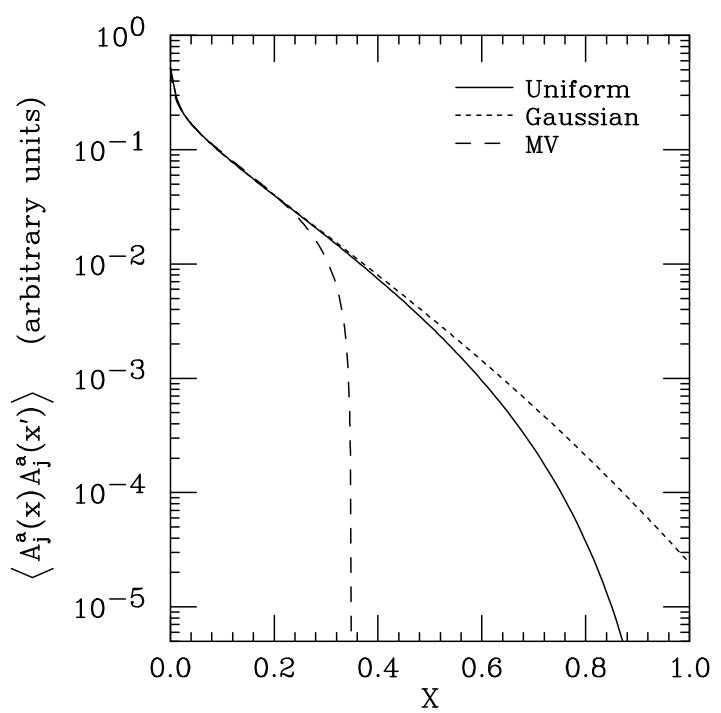

FIGURE 4. The trace of the two-point vector potential correlation function (5) in position space. The nucleon size parameters $a$ and $\Lambda_{\mathrm{QCD}}$ have been chosen so that the resulting gluon number densities match for $X \rightarrow 0$. We have fixed the longitudinal coordinates at a place where $N_{c} \chi=50 a^{-2}$. Plotted are the results of Ref. [2] (labelled "MV") as compared to Kovchegov's model [5].

wavelength gluons, where as the MV curve, generated with a hard cutoff, has the fewest.

Finally, we display the effect of the non-Abelian terms on the gluon number density. This is most easily seen on a plot of $\vec{q}^{2}$ times the number density (Fig. 6). ${ }^{3}$ From Eq. (5), it is clear that the magnitude of $N_{c} \chi$ governs the importance of these contributions. In Fig. 6 we have taken $N_{c} \chi$ to be $0,50 a^{-2}$, and $100 a^{-2}$. What we see in this plot is a transfer of gluons from low values of $\vec{q}$ to higher ones. In fact, it is possible to show that the area under this plot is conserved [3], and that this shifing of gluons from one energy to another is the only effect of the non-Abelian contributions.

In conclusion, we see that imposing a color-neutrality condition on the nucleons eliminates the divergent infrared behavior of the two-point vector potential correlation function in the MV model. Because we have obtained a well-defined expression for the gluon number density, we are able to perform a quantitative investigation of the features of the MV model. For example, we have shown that the MV model predicts a gluon number density which is proportional to $1 / q^{+}$to all orders in the

3) The fact that this curve approaches a constant for large $\vec{q}^{2}$ demonstrates the $1 / \vec{q}^{2}$ behavior described in the text. 


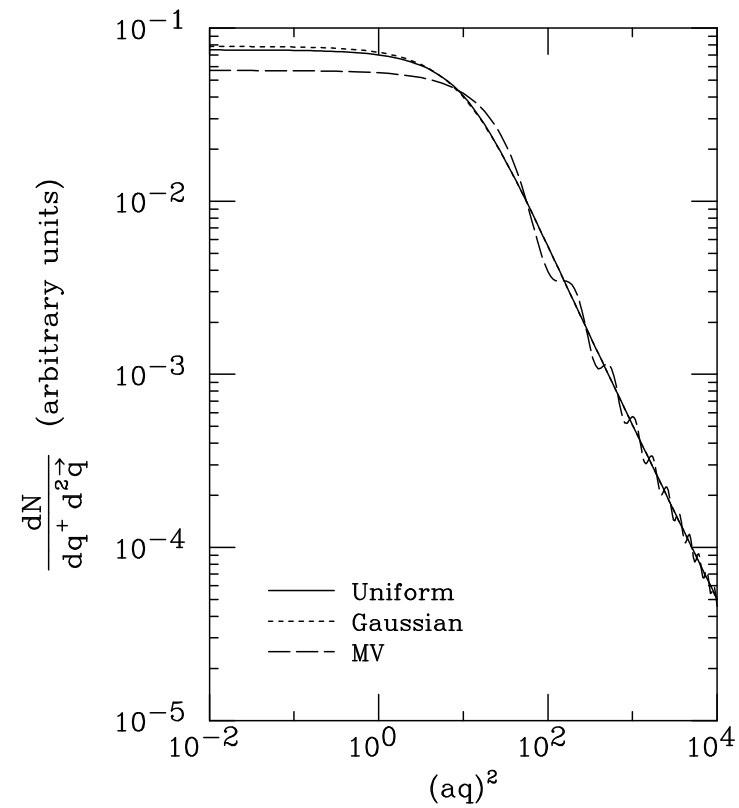

FIGURE 5. Plot of the gluon number density at fixed $q^{+}$. The nucleon size parameters $a$ and $\Lambda_{\mathrm{QCD}}$ have been fixed so that these functions match for $\vec{q}^{2} \rightarrow \infty$. We have chosen $N_{c} \chi=50 a^{-2}$. Plotted are the results of Ref. [2] (labelled "MV") as well as results using Kovchegov's model [5].

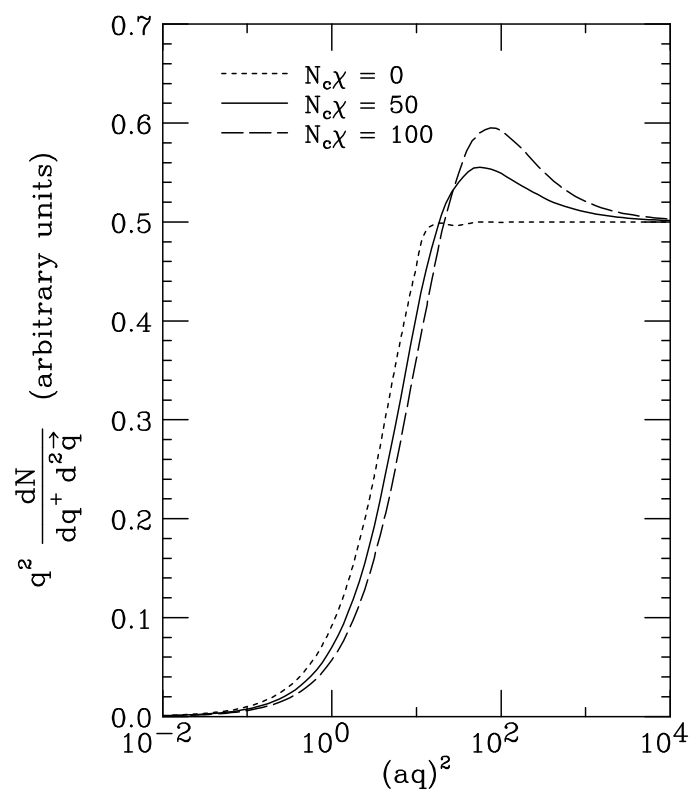

FIGURE 6. Plot of $\vec{q}^{2}$ times the gluon number density at fixed $q^{+}$for various values of the color charge density within the uniform version of Kovchegov's model [5]. As $\chi$ increases, the importance of the non-Abelian contributions increases. Conversely, $\chi \rightarrow 0$ corresponds to the Abelian limit.

charge density. We are able to compute the gluon structure function in this framework, including its absolute normalization. The details of these and other related results may be found in Ref. [3].

\section{REFERENCES}

1. L. McLerran and R. Venugopalan, Phys. Rev. D49, 2233 (1994); D49, 3352 (1994); D50, 2225 (1994); A. Ayala, J. Jalilian-Marian, and L. McLerran, Phys. Rev. D52, 2935 (1995).

2. J. Jalilian-Marian, A. Kovner, L. McLerran, and H. Weigert, Phys. Rev. D55, 5414 (1997).

3. C.S. Lam and G. Mahlon, "Color Neutrality and the Gluon Distribution in a Very Large Nucleus," hep-ph/9907281.

4. A.H. Mueller in Frontiers in Particle Physics, Cargese 1994, edited by M. Levy, J. Iliopoulos, R. Gastmans, and J.-M. Gerard, NATO Advanced Study Institute Series B, Physics, Vol. 350, (Plenum Press, 1995).

5. Yu. Kovchegov, Phys. Rev. D54, 5463 (1996).

6. L. McLerran and R. Venugopalan, Phys. Lett. B424, 15 (1998). 